\title{
Incidental Periventricular White Matter Hyperintensities Revisited: What Detailed Morphologic Image Analyses Can Tell Us
}

S oon after the introduction of MR imaging, periventricular hyperintensities started to vex the scientific community. ${ }^{1}$ While they may be a consequence of distinct disorders such as multiple sclerosis causing focal periventricular lesions or increased intraventricular pressure giving rise to extending hyperintense rims around the lateral ventricles, most periventricular white matter hyperintensities (PVWMH) constitute an unexpected or incidental finding similar to hyperintensities in the deep white matter (DWMH). ${ }^{2}$ Thus, numerous studies have attempted to clarify the etiology and clinical consequences of these signal abnormalities and their association with one another. These investigations often led to quite contradictory results which is not fully surprising given the variability with which PVWMH and DWMH have been quantified up to combining signal abnormalities at both locations in 1 rating scale. ${ }^{3,4}$ Observed anatomic overlap and a close correlation of PVWMH and DWMH volumes also led to the suggestion that their separation was arbitrary and not warranted, ${ }^{5}$ despite evidence for histopathologic differences. ${ }^{6,7}$ Still others completely dismissed linear areas of PVWMH as artifacts, ${ }^{8}$ contributing to the uncertainty of how to deal with this frequently noted phenomenon.

After a period of stagnation, the work of Valdés Hernández et al, "Morphologic, Distributional, Volumetric, and Intensity Characterization of Periventricular Hyperintensities," now promises to move the field forward again. Using MRI obtained from a large cohort of community-dwelling subjects without dementia, these investigators undertook accurately quantifying the maximum intensity and width of hyperintense signal changes observed with different categories of PVWMH, such as a thin white line, a thick rim, or penetrating toward or confluent with DWMH. ${ }^{10}$ In parallel, they also generated $3 \mathrm{D}$ reconstructions of all white matter hyperintensities (WMH). These showed that PVWMH were clearly discontinuous with DWMH in all categories, except for rare instances with PVWMH penetrating toward or confluent with DWMH. As would be expected, maximum signal intensity and width increased with the semiquantitative classification of PVWMH severity, and this change was accompanied by an increase in the prevalence of hypertension, hypercholesterolemia, and neuroradiologic evidence of stroke.
Neither these observations nor the close correlation of PVWMH widths and signal intensities with WMH volumes confirms or refutes speculations on a common pathomechanism for PVWMH and DWMH. Presented findings, however, clearly substantiate the prevailing morphologic separation of PVWMH and DWMH and indicate the availability of relatively simple and robust tools for their more refined assessment. This should open new avenues, especially for longitudinal evaluations. Furthermore, the fact that a close correlation between WMH volume and mean intensity and width of the signal was also seen for linear PVWMH strongly disputes a mainly artifactual genesis of such signal changes.

Valdés Hernández et $\mathrm{al}^{9}$ also call attention to another finding that should be followed up. In approximately $10 \%$ of investigated individuals, the authors observed "dirty" or ill-defined subtle, pale changes in the periventricular white matter, which they termed "subtle extended PVWMH." This category of periventricular signal abnormality was characterized by ill-defined boundaries and multiple small intensity peaks, which were significantly lower than those observed in all other categories. Likewise there was also no association between mean intensity and width of the signal and WMH volume seen with this category of PVWMH. Thus far "dirty white matter" on proton-attenuation or T2weighted (FLAIR) images has received attention primarily in MS, in which it appears to indicate subtle tissue damage either as a consequence of the inflammatory process or secondary to degeneration. ${ }^{11} \mathrm{Ge}$ et $\mathrm{al}^{12}$ thought that such areas might indicate prelesional changes.

In this context, it is interesting that a more recent study investigating morphologic factors related to the subsequent growth of DWMH also described subtle FLAIR hyperintensities of the brain tissue as one of the indicators of subsequent lesion development and expansion. ${ }^{13}$ Notwithstanding the superior sensitivity and undoubted contribution of quantitative techniques like diffusion tensor and magnetization transfer imaging for assessing microstructural tissue changes, these observations indicate that even conventional images may contain valuable information regarding the identification of individuals with a high probability of showing progression of white matter changes. Attention to subtle tissue 
changes on FLAIR may thus constitute another possibility for improving our insight into PVWMH development and help in selecting individuals for whom the institution of preventive therapeutic strategies might be most timely. ${ }^{14}$ Clearly this hypothesis will need to be validated by longitudinal studies.

\section{REFERENCES}

1. Zimmerman RD, Fleming CA, Lee BC, et al. Periventricular hyperintensity as seen by magnetic resonance: prevalence and significance. AJR Am J Roentgenol 1986;146:443-50

2. Brant-Zawadzki M, Fein G, Van Dyke C, et al. MR imaging of the aging brain: patchy white-matter lesions and dementia. AJNR Am J Neuroradiol 1985;6:675-82

3. Mäntylä R, Erkinjuntti T, Salonen $\mathrm{O}$, et al. Variable agreement between visual rating scales for white matter hyperintensities on MRI: comparison of 13 rating scales in a poststroke cohort. Stroke 1997;28:1614-23

4. Kapeller $\mathrm{P}$, Barber R, Vermeulen RJ, et al. Visual rating of age-related white matter changes on magnetic resonance imaging: scale comparison, interrater agreement, and correlations with quantitative measurements. Stroke 2003;34:441-45

5. DeCarli C, Fletcher E, Ramey V, et al. Anatomical mapping of white matter hyperintensities (WMH): exploring the relationships between periventricular WMH, deep WMH, and total WMH burden. Stroke 2005;36:50-55

6. Gouw AA, Seewann A, van der Flier WM, et al. Heterogeneity of small vessel disease: a systematic review of MRI and histopathology correlations. J Neurol Neurosurg Psychiatry 2011;82:126-35
7. Schmidt R, Schmidt $\mathrm{H}$, Haybaeck J, et al. Heterogeneity in age-related white matter changes. Acta Neuropathol 2011;122:171-85

8. Payne ME, Fetzer DL, MacFall JR, et al. Development of a semiautomated method for quantification of MRI gray and white matter lesions in geriatric subjects. Psychiatry Res 2002;115:63-77

9. Valdés Hernández MD, Piper RJ, Bastin ME, et al. Morphologic, distributional, volumetric, and intensity characterization of periventricular hyperintensities. AJNR Am J Neuroradiol 2014;35: $55-62$

10. Fazekas F, Chawluk JB, Alavi A, et al. MRI signal abnormalities at $\mathbf{1 . 5}$ $\mathrm{T}$ in Alzheimer's dementia and normal aging. AJR Am J Roentgenol 1987;149:351-56

11. Filippi M, Rocca MA. Dirty-appearing white matter: a disregarded entity in multiple sclerosis. AJNR Am J Neuroradiol 2010;31:390-91

12. Ge Y, Grossman RI, Babb JS, et al. Dirty-appearing white matter in multiple sclerosis: volumetric MR imaging and magnetization transfer ratio histogram analysis. AJNR Am J Neuroradiol 2003;24:1935-40

13. de Groot M, Verhaaren BF, de Boer R, et al. Changes in normalappearing white matter precede development of white matter lesions. Stroke 2013;44:1037-42

14. Schmidt $\mathrm{R}$, Berghold A, Jokinen $\mathrm{H}$, et al. White matter lesion progression in LADIS: frequency, clinical effects, and sample size calculations. Stroke 2012;43:2643-47

F. Fazekas

Department of Neurology Medical University Graz

Graz, Austria

http://dx.doi.org/10.3174/ajnr.A3714 